\title{
Chronic Anisakiasis of the Ascending Colon Associated with Carcinoma
}

\author{
Sho Mineta ${ }^{1}$, Kimiyoshi Shimanuki ${ }^{1}$, Atsushi Sugiura ${ }^{1}$, Yoshikazu Tsuchiya ${ }^{1}$, \\ Masahiro Kaneko', Yoshihiko Sugiyama ${ }^{1}$, Koho Akimaru ${ }^{3}$ and Takashi Tajiri ${ }^{3}$ \\ ${ }^{1}$ Department of Surgery, Aizu Central Hospital, Fukushima \\ ${ }^{2}$ Department of Pathology, Aizu Central Hospital, Fukushima \\ ${ }^{3}$ Surgery for Organ Function and Biological Regulation, Nippon Medical School Graduate School of Medicine
}

\begin{abstract}
Chronic anisakiasis of the colon is rare and difficult to diagnose. We report a case of chronic anisakiasis associated with advanced colonic carcinoma. A 69-year-old man was admitted for abdominal pain, diarrhea, and urticaria. Right hemicolectomy was performed because of an obstruction of the ascending colon and a palpable tumor of the right lower abdomen. The lesion was thought to be located in the deeper layers of the ascending colon. Preoperative examinations failed to detect the coexistence of anisakiasis and carcinoma of the colon. The anisakis was identified morphologically in the intestinal wall of the resected specimen and by an elevated titer of an $\operatorname{IgE}$ antibody specific to the parasite. Seventy-five cases of colonic and rectal anisakiasis, including the present case, have been reported in Japan. This is the only reported case of anisakiasis to appear in association with colonic carcinoma.
\end{abstract}

(J Nippon Med Sch 2006; 73: 169-174)

Key words: colon cancer, anisakiasis

\section{Introduction}

Anisakiasis is a parasitic disease caused by ingestion of Anisakis larvae in infested raw seafood, particularly raw fish. Though actually somewhat rare, anisakiasis is a well-known cause of gastrointestinal disease in humans. The first case was described in the Netherlands in $1960^{1}$. The most common sites of involvement in Japan, where gastrointestinal anisakiasis is frequent, are the stomach and small intestine. Since the first report of a Japanese case in 1964, Japan has become a major endemic area with more than 12,000 documented cases $^{2}$. The colon has been the site of involvement in only a very small number of these reports. Chronic anisakiasis of the colon is rare and difficult to diagnose. Patients with chronic anisakiasis typically complain of intermittent feelings of ill health and abdominal pain lasting from several weeks to 1 month. Most cases are initially misdiagnosed as another intestinal disease. Anisakiasis is a parasitic disease with several intestinal manifestations similar to those of Crohn's disease, intestinal tuberculosis, and neoplasms of the ileocolon or characterized by the presence of granuloma. We report a rare case of chronic anisakiasis of the ascending colon associated with colonic carcinoma, diagnosed by serological and histological findings.

Correspondence to Sho Mineta, Department of Surgery, Nippon Medical School, 1-1-5 Sendagi, Bunkyo-ku, Tokyo 113-8603, Japan

E-mail: minesho@nms.ac.jp

Journal Website (http://www.nms.ac.jp/jnms/) 

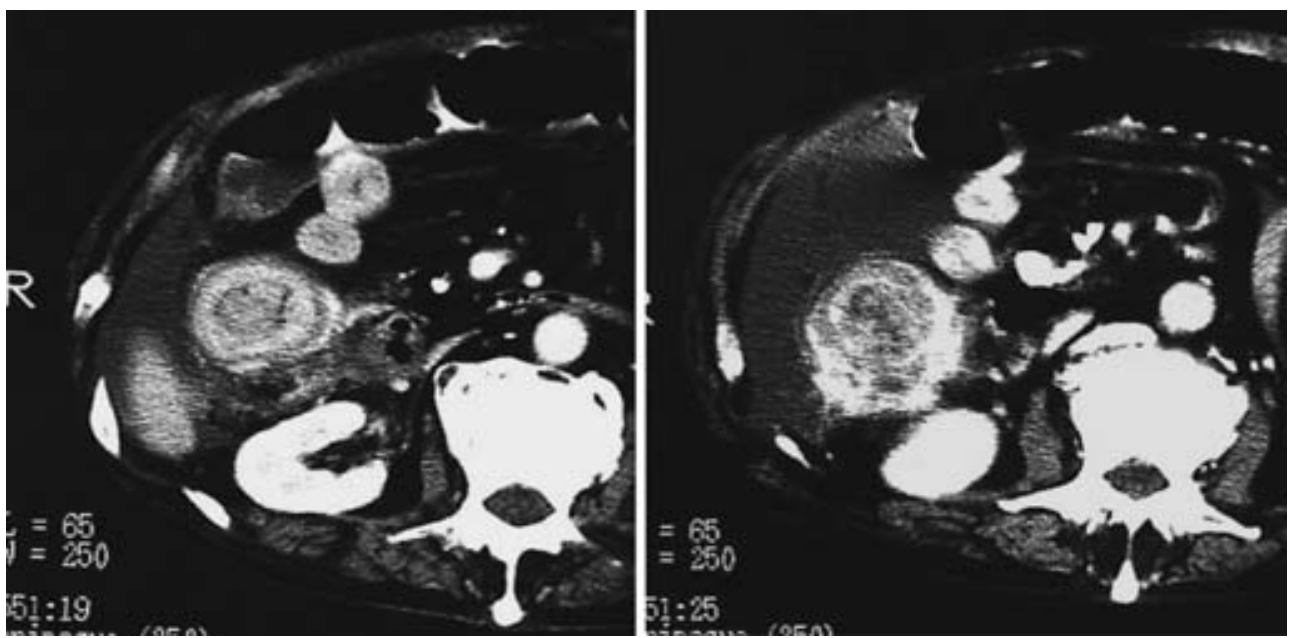

Fig. 1 Abdominal computed tomography showed a thickened colonic wall with an elevated lesion.

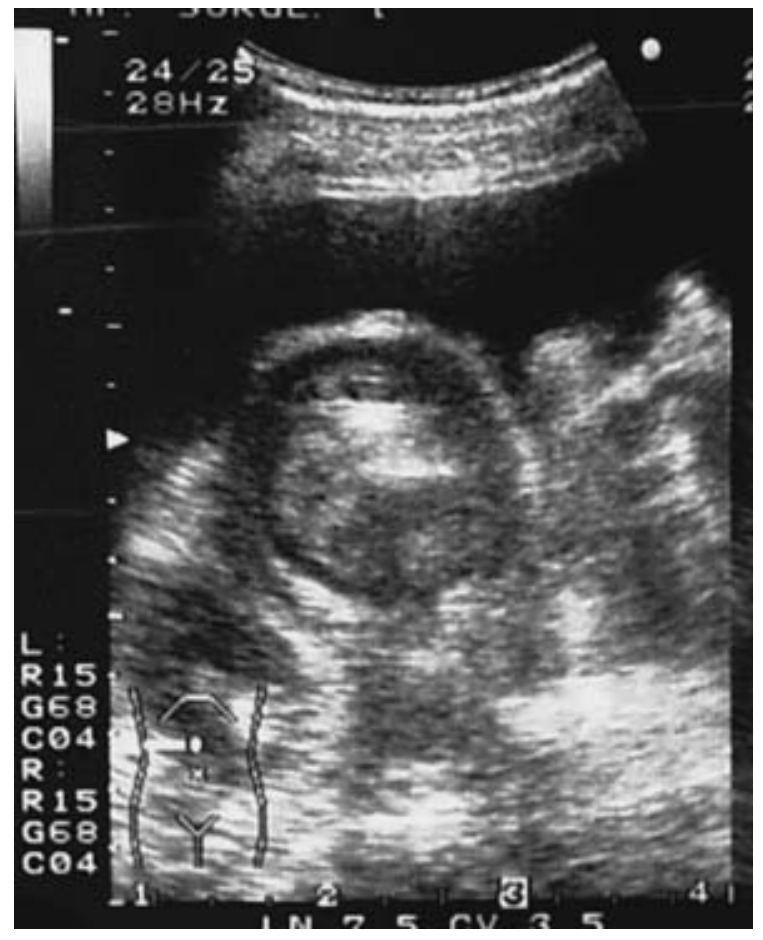

Fig. 2 Abdominal ultrasonography revealed massive ascites. Sonograms showed a thickened bowel wall and an elevated intraluminal lesion. No irregularity was found in serous layer of the colon.

\section{Case Report}

A 69-year-old man was admitted to our hospital because of right lower abdominal quadrant pain for the previous 2 weeks, vomiting, watery stool, generalized abdominal bloating, and fever. He had no notable history except for urticaria several years earlier. A palpable abdominal mass with tenderness in the right lower quadrant and ascites were noted on clinical examination. By his own account, he ate sashimi (sliced raw fish) almost every day . Abdominal roentgenograms demonstrated a dilated small-bowel loop with an air-fluid level indicating ileus. Repeated fecal examinations and washing cultures of the stool and stomach revealed no evidence of parasitic infection. Fecal occult blood testing elicited a positive reaction, and tuberculin skin testing was negative. Results of studies of the peripheral blood and biochemical markers were normal except for C-reactive protein $(28.9 \mathrm{mg} / \mathrm{d} l)$, the white blood cell count $(11,500 / \mu l)$, alkaline phosphatase (539 IU / $l$ ), and $\gamma$-glutamyl transpeptidase (185 IU/l). Hemograms showed no evidence of eosinophilia, however. Serum levels of three tumor markers-carcinoembryonic antigen, carbohydrate antigen 19-9, and alpha-fetoproteinwere all within the normal ranges.

Abdominal computed tomography showed a thickened wall of the ascending colon with an elevated lesion. Changes in the inflammatory response were also seen surrounding the large intestine (Fig. 1). Abdominal ultrasonography revealed profuse ascites. Sonograms through the ascending colon near the hepatic flexure showed a thickened bowel wall and an elevated intraluminal lesion. There was no irregularity of the serosal layer 

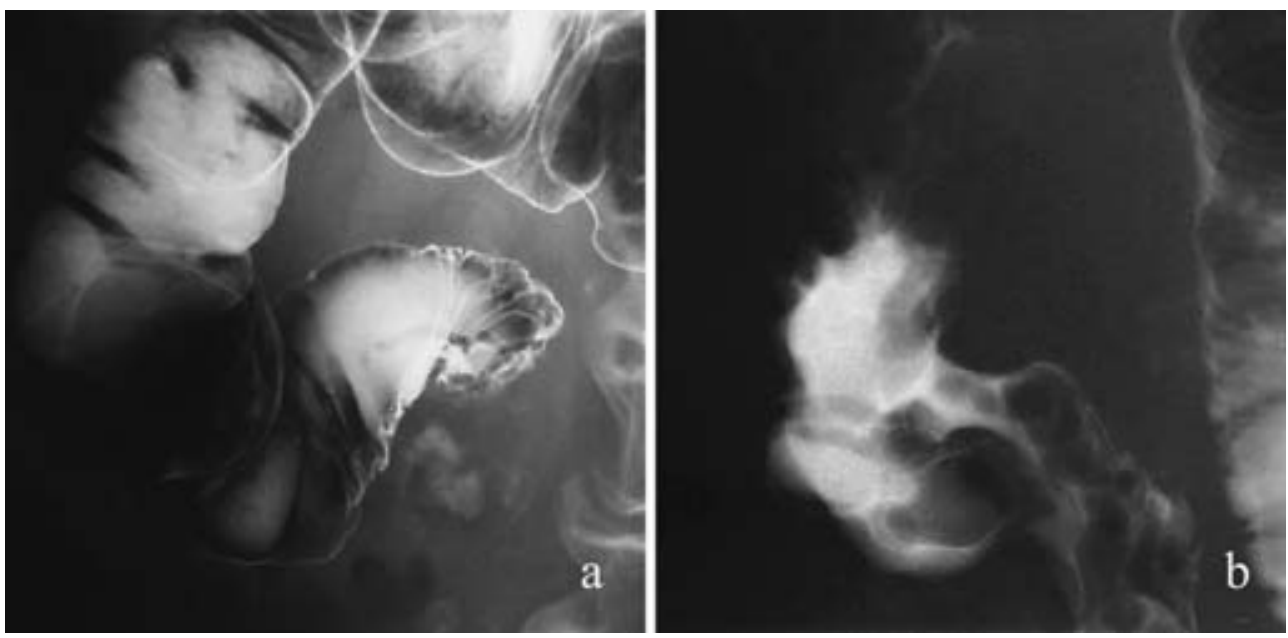

Fig. 3 A double-contrast barium-enema examination revealed obstruction of the ascending colon. The contrast medium could not pass through the ascending colon to the cecum (a). Normograde roentgenograms of the ascending colon and ileography demonstrated irregular luminal narrowing and thumb-printing (b).

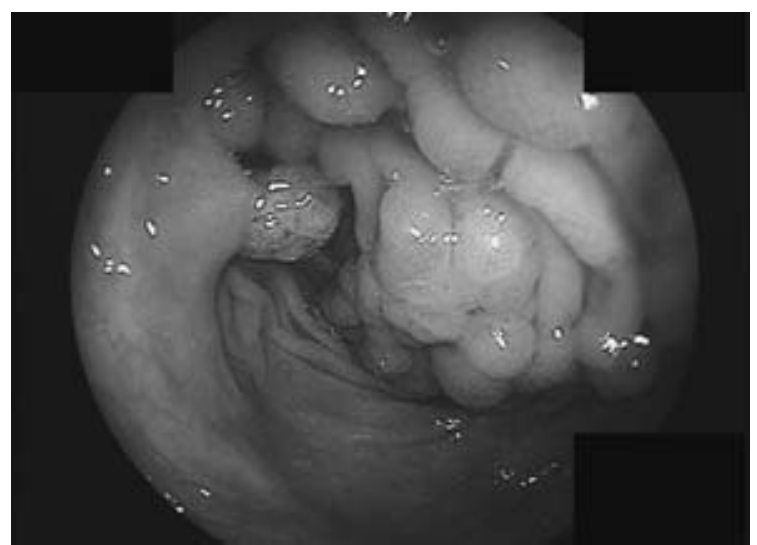

Fig. 4 Colonoscopic view of the narrowed lumen with multiple polypoid lesions, diffuse granular redness, and edematous mucosa of the ascending colon.

of the colon (Fig. 2) and no cytological evidence of malignant cells in clear, serous ascitic fluid. A double-contrast barium enema examination revealed complete obstruction of the ascending colon without visualizing the cecum (Fig. 3a). Normograde roentgenograms of the ileocecal aspect with a long tube demonstrated irregular luminal narrowing and thumb-printing with dilatation of the proximal intestin (Fig. 3b). X-ray films revealed no thread-like filling defects suggestive of a worm. The obstruction made it impossible to examine the entire colon by colonoscopy. When passed, with considerable difficulty, through the narrowed lumen, the

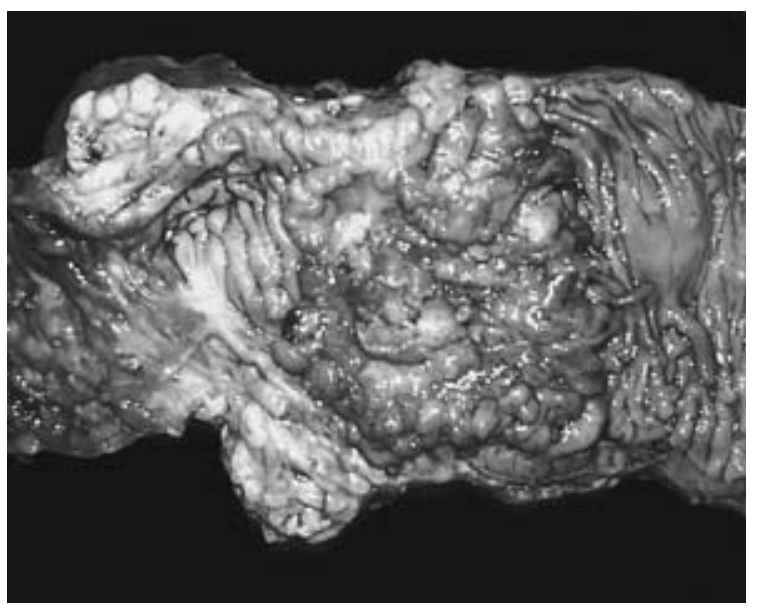

Fig. 5 The gross pathologic specimen showed an advanced carcinoma with multiple polypoid lesions.

colonoscope revealed multiple polypoid lesions with diffuse red granular and edematous mucosa. It failed, however, to show the presence of the worm. Adenocarcinoma was found in a biopsy specimen from a polypoid lesion in the narrow and rigid segment (Fig. 4). The patient underwent right hemicolectomy, with lymph node dissection because of the suspected invasion of the tumor into the deeper layers of the ascending colon. Intraoperative cytological examination of the ascites fluid revealed no malignant cells. Gross pathologic examination of the resected specimen (Fig. 5) showed advanced carcinoma of the ascending colon with multiple 

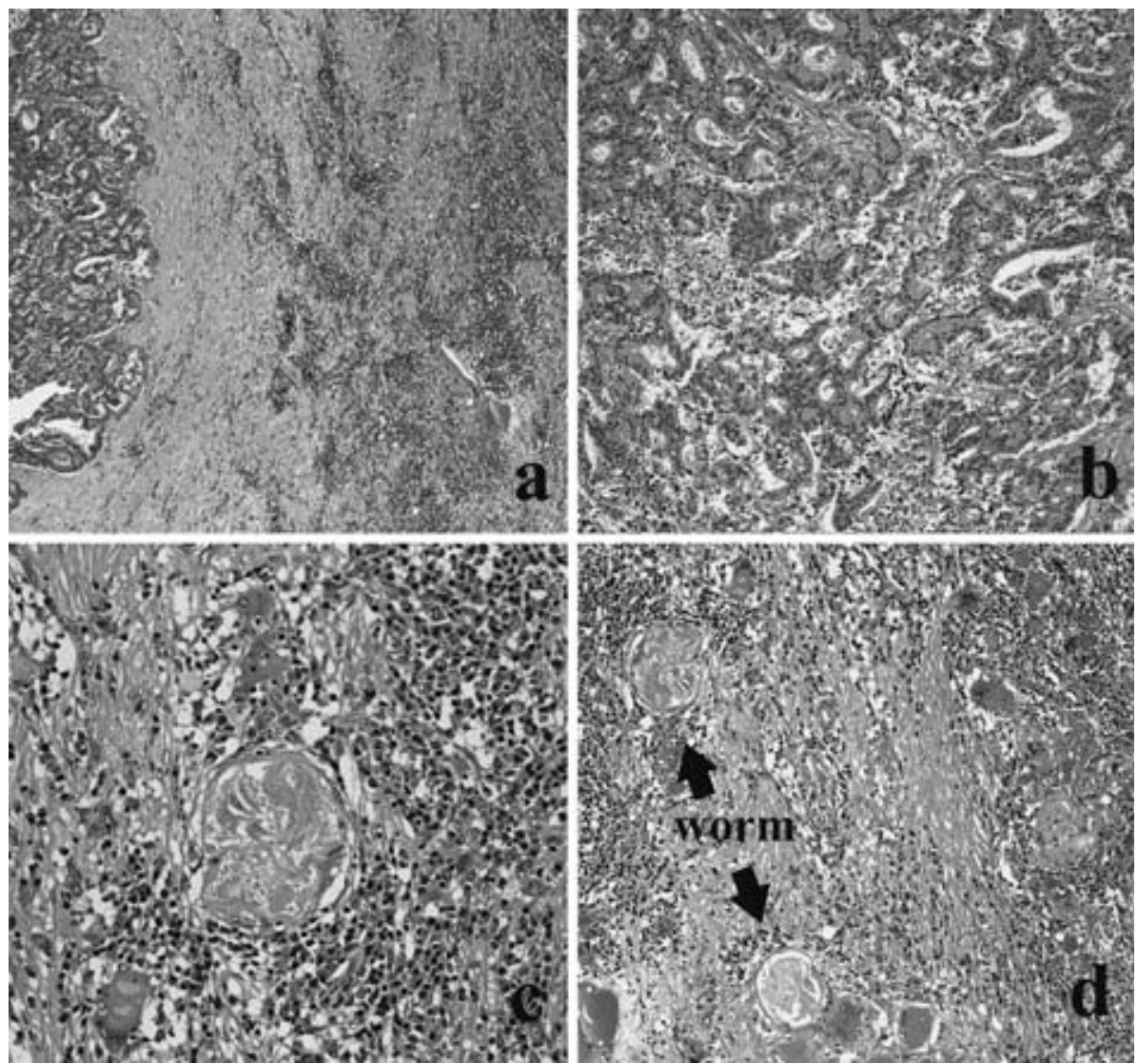

Fig. 6 Histological studies revealed phlegmonous inflammation of the bowel wall and well-differentiated adenocarcinoma (a)(b). The cross section of a worm was found within the edematous submucosal layer. A structure close to the Y-shaped form was discerned (c). The granulomatous lesion was composed of foreign-body giant cells, lymphocytes, and neutrophils. The Splendore-Hoeppli phenomenon was seen $(\mathrm{d})$.

polypoid lesions. Histological examination revealed phlegmonous inflammation of the bowel wall with well-differentiated adenocarcinoma infiltrating the subserosal layer (Fig. 6a, 6b). The cross section of a worm was found within the strongly edematous submucosal layer. A structure similar to the Yshaped lateral cord characteristic of the Anisakis larva was observed, but only faintly due to the degeneration of the larva (Fig. 6c). The granulomatous site was composed of foreign-body giant cells, lymphocytes, and neutrophils. An amorphous and eosinophilic substance surrounding the degenerated larva, a feature typical of the socalled Splendore-Hoeppli phenomenon ${ }^{3}$, was also observed at the same site. (Fig. 6d). Preoperative findings were not sufficient to differentiate between advanced colonic carcinoma and anisakiasis in this case. Total and Anisakis-specific IgE antibodies were positive in the patient's serum $(54.5 \mathrm{UA} / \mathrm{ml}$; standard value $0.34 \mathrm{UA} / \mathrm{ml}$ ) after the operation, although $\operatorname{IgM}$ and $\operatorname{IgA}$ antibodies to Anisakis were negative in a postoperative enzyme-linked immunosorbent assay. The final diagnosis was based on elevated specific $\operatorname{IgE}$ antibody titers and identification of the parasites morphologically in the intestinal wall of the surgical specimen.

\section{Discussion}

Anisakiasis most commonly involves the wall of the stomach and small intestine, causing an inflammatory response and acute symptoms, such as sudden abdominal pain and allergic reaction. Only rarely is the infection located in the colon. The most reasonable explanation for the rarity of colonic infection is the considerable distance to the colon-a 
distance generally too far for the orally ingested larvae to travel. Of the 4,682 cases of anisakiasis reported in Japan from 1965 through 1987, 4,296 cases were gastric . Intestinal and extragastrointestinal anisakiasis is far rarer, accounting for 375 and 11 cases, respectively ${ }^{4}$. Later reviews have reported an accumulated total of as many as 30,000 cases of anisakiasis in Japan by 1997 and 75 cases of colonic anisakiasis by 2001. Among the 75 reported cases of colonic infection, the infection sites were the cecum in 21 cases, the ascending colon in 25 cases, the transverse colon in 15 cases, the descending colon in 5 cases, the sigmoid colon in 4 cases, the rectum in 4 cases, and unknown sites in 2 cases. More than half of the cases of colonic anisakiasis were on the right side. Colectomy was performed in 12 cases, including 5 cases of infection in the ascending colon, 2 in the sigmoid colon, 3 in the transverse colon, and 2 in the cecum. Our case of chronic anisakiasis associated with carcinoma in the colon is the first reported case of this type.

The acute form of anisakiasis presents with severe abdominal pain and anaphylactoid reactions after the consumption of Anisakis. Anisakiasis in the acute phase can usually be diagnosed immunologically by the presence of antibodies specific to Anisakis larvae and a recent history of raw fish consumption. The condition is easily diagnosed if the body of a worm is confirmed by an endoscopic examination or barium enema study of the large intestine. Clinical features of infection with Anisakis are often similar to those of acute appendicitis, intestinal occlusion, and Crohn's disease. There is no anthelmintic agent for the treatment of anisakiasis. In acute cases of the stomach or colon, endoscopic removal of the larva provides a complete cure.

Cases of chronic anisakiasis are quite difficult to diagnosis solely on the basis of pathologic examination or immunological testing. Antibody titers may decrease during chronic periods, and the body of a worm is difficult to identify once it begins to degenerate. Patients with chronic anisakiasis typically have symptoms of vomiting, diarrhea, mild abdominal pain, and chronic urticaria. Most cases are diagnosed as abdominal tumors and intestinal obstruction caused by the granuloma formation around the larvae. The $\operatorname{IgE}$ antibody specific to Anisakis is positive in nearly half of the patients with chronic urticaria ${ }^{5}$. Serologic tests are more important in chronic cases due to the unreliability of diagnosis by endoscopic examination. The diagnosis is confirmed by the detection of $\operatorname{IgG}$ and $\operatorname{IgA}$ antibodies specific to Anisakis larvae in blood samples. This test can be positive from day 1 after ingestion of the larva and becomes negative at between 6 and 12 months. The larva itself typically dies within 8 weeks. In chronic cases, however, specific $\operatorname{IgE}$ antibodies mobilize against the organism and the levels of total $\mathrm{IgE}$ rise $^{6}$. Gomez et $\mathrm{al}^{7}$ have demonstrated the presence of $\operatorname{IgE}$ antibodies against Anisakis simplex in $80 \%$ of patients with anisakiasis. The accuracy of this examination is open to question, however, as serum IgE antibodies against A. simplex have also been found in $10 \%$ of healthy individuals in Japan ${ }^{8}$. The blood eosinophil count usually remains normal in chronic anisakiasis.

The differential diagnosis of the coexisting inflammatory granular disease in chronic cases includes acute appendicitis, neoplasms , intussusception, diverticulitis, ileus, food poisoning, ischemic colitis, intestinal tuberculosis, and Crohn's disease. The differentiation from anisakiasis by clinical, roentgenological, and endoscopic approaches is difficult under these circumstances, leaving biopsy as the only option. Intestinal or colonic anisakiasis is occasionally diagnosed only after surgical exploration. When surgery is performed, histological sections show pathologic features that differ according to the stage of the disease and the location of the larvae. In earlier stages the features of the worm may be identifiable from an eosinophilic granuloma. Identification becomes more difficult in the later stages, when the worm degenerates into fragments of abscessed tissue. When the larva is only slightly degenerated, the condition can be diagnosed easily and accurately by identifying the Y-shaped lateral cords characteristic of the worm³.

Differential diagnosis is very difficult when a chronic anisakiasis and a cancer lesion are merged. In attempting a differential diagnosis, physicians 
should consider factors such as the fish consumption of the patient in relation to the onset of symptoms. If a patient has allergic symptoms such as urticaria, a symptom in our case, the physician should inquire about the patient's eating habits, ascertain when the present illness began, and consider the possibility of anisakiasis. Urticaria is an important indicator of the potential presence of anisakiasis 9 . Abundant ascites was found in our case. Ascitic fluid can be found in varying amounts in intestinal anisakiasis, as well. Results of preoperative imaging studies were too indistinct to reveal whether the ascites was produced from the inflammatory process of anisakiasis or from an extraluminal invasion of carcinoma. The cytologic examination of ascitic fluid, on the other hand, can be helpful for differential diagnosis. Surgery is mandatory in chronic anisakiasis of the gastrointestinal tract with mass formation and intestinal stenosis.

\section{References}

1. Van Thiel PH, Kuipers FC, Roskam RTH: A nematode parasite to herring, causing acute abdominal syndromes in man. Trop Geogr Med 1960; 2: $97-113$.

2. Ishikura H, Kikuchi $\mathrm{K}$, Nagasawa $\mathrm{K}$, et al.:
Anisakidae and anisakidosis. In Progress in clinical parasitology, vol. III (Sun T, ed), 1993; pp 43-102, Springer-Verlag, New York.

3. Smith JW, Wootten R: Anisakis and anisakiasis. In Advances in Parasitology, vol 16 (Baker JR, ed), 1978; pp 93-163, Academic, London, New York, San Francisco.

4. Ishikura H, Kobayashi $\mathrm{Y}$, Miyamoto $\mathrm{K}$, et al.: Transition of occurrence of anisakiasis and its paratenic host fishes in Japan, with pathogenesis of anisakiasis. (in Japanese) Hokkaido Igaku Zasshi 1988; 63: 376-391.

5. Hirai S: Anisakis, a relevant etiologic factor in acute and chronic urticaria- significant detection of Anisakis specific $\operatorname{IgE}$ in acute and chronic urticaria. (in Japanese) Arerugi no Rinsho 2000; 20: 18-25.

6. Eskesen A, Strand EA, Andersen SN, Rosseland A, Hellum KB, Oystein A: Strand Anisakiasis presenting as an obstructive duodenal tumor. A Scandinavian Case. Scand J Infect Dis 2001; 33: 7576.

7. Gomez B, Tabar AI, Tunon T, et al.: Eosinophilic gastroenteritis and anisakis. Allergy 1998; 53: 11481154.

8. Takabe K, Ohki S, Kunihiro O, et al.: Anisakidosis: a cause of intestinal obstruction from eating sushi. Am J Gastroenterol 1998; 93: 1172-1173.

9. Muraoka A, Suehiro I, Fujii M, et al.: Acute gastric anisakiasis 28 cases during the last 10 years. Dig Dis Sci 1996; 41: 2362-2365.

(Received, January 27, 2006)

(Accepted, April 10, 2006) 